\title{
Augmenting and preserving partition connectivity of a hypergraph
}

\author{
Xiaofeng Gu and Hong-Jian Lai
}

\begin{abstract}
Let $k$ be a positive integer. A hypergraph $H$ is $k$-partition-connected if for every partition $P$ of $V(H)$, there are at least $k(|P|-1)$ hyperedges intersecting at least two classes of $P$. In this paper, we determine the minimum number of hyperedges in a hypergraph whose addition makes the resulting hypergraph $k$-partition-connected. We also characterize the hyperedges of a $k$-partition-connected hypergraph whose removal will preserve $k$-partition-connectedness.
\end{abstract}

\section{The problem}

The problem of edge connectivity augmentation seems to be initiated by Watanabe and Nakamura [23], in which they investigated the minimum number of edges that must be added to a graph $G$ so that the resulting graph is $k$-edge-connected, for given integer $k$ and graph $G$. Frank [8] provided an efficient algorithm to solve this kind of problem. For connectivity augmentation in graphs and hypergraphs, two recent survey papers [12] and [21] are very informative.

Frank, Király and Kriesell [10] introduced $k$-partition-connected hypergraphs as a generalization of $k$-edge-connected hypergraphs (see Section 2 for the definition). The augmentation and preservation problems related to partition connectivity of graphs and hypergraphs have been investigated in $[9,11,13,16,15]$, among others.

Theorem 1.1. Let $G$ be a graph and $k$ be a positive integer. The following are equivalent.

(i) There exists an edge set $X$ such that $G+X$ is k-partition-connected.

(ii) (Frank and Király, a weaker statement of Theorem 5.2 of [9]) $|X| \geq$ $k(|P|-1)-e(P)$ for every partition $P$ of $V(G)$, where $e(P)$ is the number of edges whose ends are in different classes of $P$.

(iii) (Haas, Theorem 1 of [11]) $|X|=k(|V(G)|-1)-|E(G)|$ and for subgraphs $S$ of $G$ with at least two vertices, $|E(S)| \leq k(|V(S)|-1)$. 
Theorem 1.2. (Király and Makai, a weaker statement of Corollary 4.13 of [13]) Let $H$ be a hypergraph and $k$ be a positive integer. The following are equivalent.

(i) There exists a hyperedge set $X$ such that $H+X$ is $k$-partition-connected. (ii) $|X| \geq k(|P|-1)-e(P)$ for every partition $P$ of $V(H)$, where $e(P)$ is the number of hyperedges intersecting at least two classes of $P$.

Liu, Lai and Chen [16] generalize Theorem 1.1 and find the exact minimum number of edges that must be added to make the resulting graph be $k$-partition-connected.

The research in this paper is motivated by the results above. Our goal is to determine the minimum number of hyperedges in a hypergraph whose addition makes the resulting hypergraph $k$-partition-connected (Theorems 5.4 and 5.8 present the exact minimum value and a minimax formula). We also characterize the hyperedges in a $k$-partition-connected hypergraph whose removal will preserve the $k$-partition-connectedness of the hypergraph (Theorem 6.2).

Relevant definitions and preliminaries will be presented in Section 2. Undefined terms can be found in [1] for hypergraphs and [2] for graphs. In Section 3, uniformly dense hypergraphs and their relationship with partition connectivity of hypergraphs will be discussed. A few useful tools (Theorems 4.4 and 4.9) will be developed in Section 4. These tools will be applied to the studies of the augmentation and preservation problems of partition connectivity of hypergraphs in Sections 5 and 6.

\section{Notations and preliminaries}

A hypergraph $H$ is a pair $(V, \mathcal{E})$ where $V$ is the vertex set of $H$ and $\mathcal{E}$ is a collection of not necessarily distinct nonempty subsets of $V$, called hyperedges or simply edges of $H$. A single element edge is referred as a loop and two edges with the same vertices are parallel edges. We use $K_{1}$ to denote the hypergraph with one vertex and no edges. If $W \subset V$, the hypergraph $\left(W, \mathcal{E}_{W}\right)$, where $\mathcal{E}_{W}=\{F: \forall F \in \mathcal{E}$ with $F \subseteq W\}$ is a sub-hypergraph induced by the vertex subset $W$, and is denoted by $H[W]$. If $X \subseteq \mathcal{E}$ and $V_{X}=\cup_{F \in X} X$, then $\left(V_{X}, X\right)$ is defined as the sub-hypergraph induced by the edge subset $X$ and is denoted by $H[X]$. A hypergraph $H$ is nontrivial if $H$ has at least one non-loop edge.

Let $\omega(H)$ denote the number of components in $H$. For a positive integer $k$, a hypergraph $H$ is $k$-edge-connected if for every nonempty proper subset $U$ of $V(H)$, there are at least $k$ hyperedges intersecting both $U$ and 
$V(H) \backslash U$. The edge connectivity of $H$ is the maximum $k$ such that $H$ is $k$ edge-connected. A hypergraph $H$ is $k$-partition-connected if $e(P) \geq k(|P|-1)$ for every partition $P$ of $V(H)$, where $|P|$ denotes the number of classes in $P$ and $e(P)$ denotes the number of edges intersecting at least two classes of $P$. Equivalently, $H$ is $k$-partition-connected if, for any subset $X \subseteq \mathcal{E}(H)$, $|X| \geq k(\omega(H-X)-1)$. As $P$ can be any partitions of $V(H)$ into two nonempty subsets, it follows by definition that every $k$-partition-connected hypergraph must be $k$-edge-connected. Often a 1-partition-connected hypergraph is also referred as a partition-connected hypergraph. It follows from definition that a graph is partition-connected if and only if it is connected. In general, partition-connected hypergraphs must be connected, but a connected hypergraph may not be partition-connected. The partition connectivity of $H$ is the maximum $k$ such that $H$ is $k$-partition-connected.

A hypergraph $H$ is a hyperforest if for every nonempty subset $U \subseteq V(H)$, $|\mathcal{E}(H[U]) \leq| U \mid-1$. A hyperforest $T$ is a hypertree if $|\mathcal{E}(T)|=|V(T)|-1$. For a hypergraph $H$, let $\tau(H)$ be the maximum number of edge-disjoint spanning hypertrees in $H$ and $a(H)$ be the minimum number of edge-disjoint hyperforests whose union is $\mathcal{E}(H)$. For a graph $G, \tau(G)$ is the spanning tree packing number of $G$ and $a(G)$ is the arboricity of $G$.

The following theorem of Nash-Williams and Tutte shows that the $k$ partition-connectedness of a graph $G$ is equivalent to the property that $G$ has $k$ edge-disjoint spanning trees.

Theorem 2.1. (Nash-Williams [18] and Tutte [22]) Let $G$ be a connected graph and $k$ be a positive integer. Then $\tau(G) \geq k$ if and only for any $X \subseteq$ $E(G),|X| \geq k(\omega(G-X)-1)$.

Nash-Williams published a dual theorem of Theorem 2.1, characterizing graphs that can be decomposed to at most $k$ forests (Theorem 2.2).

Theorem 2.2. (Nash-Williams [19]) Let $G$ be a connected graph and $k$ be a positive integer. Then $a(G) \leq k$ if and only if for any subgraph $S$, $|E(S)| \leq k(|V(S)|-1)$.

Frank, Király and Kriesell [10] extended both results to hypergraphs.

Theorem 2.3. (Frank, Király and Kriesell [10]) Let $H$ be a hypergraph and $k$ be a positive integer. Then $\tau(H) \geq k$ if and only if for every $X \subseteq \mathcal{E}(H)$, $|X| \geq k(\omega(H-X)-1)$ (or, equivalently, $H$ is $k$-partition-connected).

By Theorem 2.3, $\tau(H)$ is the partition connectivity of $H$ and a hypertree is a minimal partition-connected hypergraph. 
Theorem 2.4. (Frank, Király and Kriesell [10]) Let $H$ be a hypergraph and $k$ be a positive integer. Then $\alpha(H) \leq k$ if and only if for any subgraph $S$, $|\mathcal{E}(S)| \leq k(|V(S)|-1)$.

Let $H_{1}, H_{2}, \ldots, H_{c}$ be the components of a hypergraph $H$ such that each $H_{i}$ has a spanning hypertree $T_{i}$ for $i=1,2, \ldots, c$. Then $\cup_{i} T_{i}$ is a hyperbase of $H$. By definition, if $H$ is connected, then a hyperbase is a spanning hypertree of $H$. Theorem 2.3 implies the following corollary.

Corollary 2.5. A hypergraph $H$ has $k$ edge-disjoint hyperbases if and only if for every $X \subseteq \mathcal{E}(H),|X| \geq k(\omega(H-X)-\omega(H))$.

\section{Uniformly dense hypergraphs}

In this section, we consider only loopless hypergraphs. Let $E$ be a hyperedge in a hypergraph $H$. By $H / E$ we denote the hypergraph obtained from $H$ by contracting the hyperedge $E$ into a new vertex $v_{0}$ and by removing resulting loops if there are any. That is, $V(H / E)=(V(H) \backslash E) \cup\left\{v_{0}\right\}$ and a hyperedge $E^{\prime} \in \mathcal{E}(H / E)$ if and only if either $E^{\prime}=E^{\prime \prime}$ for some $E^{\prime \prime} \in \mathcal{E}(H)$ with $E^{\prime \prime} \cap E=\emptyset$ or $E^{\prime}=\left(E^{\prime \prime} \backslash E\right) \cup\left\{v_{0}\right\}$ for some $E^{\prime \prime} \in \mathcal{E}(H) \backslash\{E\}$ with $E^{\prime \prime} \cap E \neq \emptyset$. The hyperedge $E^{\prime}$ is called the image of $E^{\prime \prime}$ and $E^{\prime \prime}$ is a preimage of $E^{\prime}$. If $X \subseteq \mathcal{E}(H)$, then $H / X$ is a hypergraph obtained from $H$ by contracting all edges in $X$. If $S$ is a sub-hypergraph of $H$, then $H / S$ denotes $H / \mathcal{E}(S)$.

For any nonempty subset $X \subseteq \mathcal{E}(H)$, the density of $X$ is defined to be

$$
d_{H}(X)=\frac{|X|}{|V(H[X])|-\omega(H[X])} .
$$

We often use $d(H)$ for $d(\mathcal{E}(H))$. If $X \subset \mathcal{E}(H)$, then by the definition of contraction, $d(H / X)=\frac{|\mathcal{E}(H)-X|}{|V(H / X)|-\omega(H)}$. Following [5], the strength $\eta(H)$ and the fractional arboricity $\gamma(H)$ of a nontrivial hypergraph $H$ are defined, respectively, as

$$
\eta(H)=\min \left\{\frac{|\mathcal{E}(H)-X|}{|V(H / X)|-\omega(H)}\right\}, \text { and } \gamma(H)=\max \{d(H[X])\},
$$

where the minimum and maximum are taken over all edge subsets $X \subseteq \mathcal{E}$ so that the denominators are nonzero. We adopt the convention by defining $\eta\left(K_{1}\right)=d\left(K_{1}\right)=\gamma\left(K_{1}\right)=\infty$. It follows immediately that for any loopless nontrivial hypergraph $H$,

$$
\eta(H) \leq d(H) \leq \gamma(H) .
$$


Let $H$ be a hypergraph and $t$ be a positive integer. A $t$-packing of $H$ is a family $\mathscr{F}$ of hyperbases in $H$ such that each hyperedge of $H$ is in at most $t$ members of $\mathscr{F}$. Let $\eta_{t}(H)$ denote the largest cardinality of $t$-packings of $H$. Dually, a $t$-covering of $H$ is a family $\mathscr{F}$ of hyperforests in $H$ such that each hyperedge of $H$ is in at least $t$ members of $\mathscr{F}$. Let $\gamma_{t}(H)$ denote the smallest cardinality of $t$-coverings of $H$. (If $H$ has a loop, then $\gamma_{t}(H)=\infty$.)

The proposition below follows from Theorems 2.3, 2.4 and Corollary 2.5.

Proposition 3.1. For any hypergraph $H$, each of the following holds.

(i) $\alpha(H)=\gamma_{1}(H)=\lceil\gamma(H)\rceil$.

(ii) $\eta_{1}(H)=\lfloor\eta(H)\rfloor$.

(iii) If $H$ is connected, then $\tau(H)=\eta_{1}(H)$.

Let $H=(V, \mathcal{E})$ be a hypergraph and let $t>0$ be an integer. The hypergraph $H^{(t)}=\left(V, \mathcal{E}^{\prime}\right)$ has the same vertex set $V$, where $\mathcal{E}^{\prime}$ is obtained by replacing each hyperedge in $\mathcal{E}$ by a set of $t$ parallel hyperedges.

Theorem 3.2. Let $H$ be a hypergraph and $s, t>0$ be integers. Each of the following holds.

(i) $H$ has a $t$-packing of cardinality $s$ if and only if $\eta(H) \geq s / t$.

(ii) $H$ has a t-covering of cardinality $s$ if and only if $\gamma(H) \leq s / t$.

(iii) $\eta_{t}(H)=\lfloor t \eta(H)\rfloor$ and $\gamma_{t}(H)=\lceil t \gamma(H)\rceil$.

Proof. It suffices to prove (i) and (ii).

(i) $H$ has a $t$-packing of cardinality $s$ if and only if $H^{(t)}$ has $s$ edge-disjoint hyperbases. By Proposition 3.1, this is equivalent to $\eta\left(H^{(t)}\right) \geq \eta_{1}\left(H^{t}\right) \geq s$. By definition, it is equivalent to $t \eta(H) \geq s$, or $\eta(H) \geq s / t$.

(ii) $H$ has a $t$-covering of cardinality $s$ if and only if $H^{(t)}$ can be decomposed into $s$ hyperforests. By Proposition 3.1, this is equivalent to $\gamma\left(H^{(t)}\right) \leq$ $s$. By definition, it is equivalent to $t \gamma(H) \leq s$, or $\gamma(H) \leq s / t$.

A hypergraph $H$ is uniformly dense if $d(H)=\gamma(H)$. The next result extends Theorem 6 of [5].

Theorem 3.3. Let $H$ be a hypergraph. The following are equivalent.

(i) $\eta(H)=\gamma(H)$.

(ii) $\eta(H)=d(H)$.

(iii) $d(H)=\gamma(H)$.

(iv) There is a family $\mathscr{F}$ of hyperbases of $H$ and a positive integer $t$ such that $\mathscr{F}$ is both a t-packing and a t-covering.

Proof. (i) $\Rightarrow$ (ii) and (i) $\Rightarrow$ (iii) follow from (2).

(ii) $\Rightarrow$ (iv): Suppose that $\eta(H)=d(H)=\frac{h}{t}$ for some integers $h, t>0$. By Theorem 3.2 (iii), $h=t \eta(H)=\eta_{t}(H)$, and so $H$ has a family $\mathscr{F}=$ 
$\left\{T_{1}, T_{2}, \ldots, T_{h}\right\}$ of hyperbases such that every hyperedge $E \in \mathcal{E}(H)$ is in at most $t$ members of $\mathscr{F}$. As $\eta(H)=d(H)$, we have $t \eta(H)(|V(H)|-\omega(H))=$ $\eta_{t}(H)(V(H)-\omega(H))=\sum_{i=1}^{h}\left|T_{i}\right| \leq t|\mathcal{E}(H)|=t \eta(H)(|V(H)|-\omega(H))$, and so every hyperedge of $H$ is in exactly $t$ members of $\mathscr{F}$. Thus (iv) holds.

(iii) $\Rightarrow\left(\right.$ iv): Let $g \geq t>0$ be integers such that $d(H)=\gamma(H)=\frac{g}{t}$. By Theorem 3.2 (iii), $g=t \gamma(H)=\gamma_{t}(H)$, and so $H$ has a family $\mathscr{F}=$ $\left\{B_{1}, B_{2}, \ldots, B_{h}\right\}$ of hyperforests such that every hyperedge $E \in \mathcal{E}(H)$ is in at least $t$ members of $\mathscr{F}$. As $\eta(H)=d(H)$, we have $t \gamma(H)(|V(H)|-\omega(H))=$ $\gamma_{t}(H)(V(H)-\omega(H)) \geq \sum_{i=1}^{g}\left|B_{i}\right| \geq t|\mathcal{E}(H)|=t \gamma(H)(|V(H)|-\omega(H))$, and so each $B_{i}$ is a hyperbase of $H$ for $1 \leq i \leq g$; and every hyperedge of $H$ is in exactly $t$ members of $\mathscr{F}$. Thus (iv) holds.

(iv) $\Rightarrow(\mathrm{i})$ : Since $\mathscr{F}$ is a $t$-packing as well as a $t$-covering of cardinality $s$, by Theorem 3.2, $\eta(H) \geq \frac{s}{t} \geq \gamma(H) \geq \eta(H)$. Thus (i) holds.

Lemmas 3.4 and 3.5 will be needed in the next section.

Lemma 3.4. Let $H$ be a nontrivial hypergraph and $l \geq 1$ be a fractional number. Then each of the following holds.

(i) If $X \subseteq \mathcal{E}(H)$, then $\eta(H) \leq \eta(H / X)$.

(ii) If $X \subseteq \mathcal{E}(H)$ and $\eta(H[X])>\eta(H)$, then $\eta(H / X)=\eta(H)$.

(iii) If $d(H) \geq l$, then there exists a nonempty subset $X \subseteq \mathcal{E}(H)$ such that $\eta(H[X]) \geq l$.

Proof. (i) By definition, there exists $Y^{\prime} \subseteq \mathcal{E}(H / X)$ such that $\eta(H / X)=$ $d\left((H / X) / Y^{\prime}\right)$. Let $Y \subseteq \mathcal{E}(H)$ be a preimage of $Y^{\prime}$. Then $\eta(H / X)=$ $d\left((H / X) / Y^{\prime}\right)=d(H /(X \cup Y)) \geq \eta(H)$. (If $H[X]$ is spanning, then $\eta(H / X)=\infty$.)

(ii) It suffices to show that $\eta(H) \geq \eta(H / X)$. By definition, there exists a nonspanning subset $T$ of $\mathcal{E}(H)$ such that $\eta(H)=d(H / T)=\frac{|\mathcal{E}(H) \backslash T|}{|V(H / T)|-\omega(H)}$. We use $X^{c}$ to denote $\mathcal{E}(H) \backslash X$ and let $X \cap T=T_{1}$ and $X^{c} \cap T=T_{2}$. Then

$$
\eta(H)=\frac{\left|X \backslash T_{1}\right|+\left|X^{c} \backslash T_{2}\right|}{|V(H / T)|-\omega(H)} .
$$

If $V\left(H\left[T_{1}\right]\right)=V(H[X])$, then let $T_{2}^{\prime} \subseteq \mathcal{E}\left(H / T_{1}\right)$ be the image of $T_{2}$. By definition, $\eta(H / X)=\eta\left(H / T_{1}\right) \leq d\left(\left(H / T_{1}\right) / T_{2}^{\prime}\right)=d(H / T)=\eta(H)$. Therefore, we assume that $V\left(H\left[T_{1}\right]\right) \neq V(H[X])$. By definition, $\eta(H[X]) \leq$ $d\left(H[X] / T_{1}\right)=\frac{\left|X \backslash T_{1}\right|}{\left|V\left(H[X] / T_{1}\right)\right|-\omega(H[X])}$. As $\eta(H[X])>\eta(H)$, we have

$$
\left|X \backslash T_{1}\right|>\eta(H)\left(\left|V\left(H[X] / T_{1}\right)\right|-\omega(H[X])\right) .
$$


By (3) and (4),

(5) $\eta(H)\left(|V(H / T)|-\omega(H)-\left|V\left(H[X] / T_{1}\right)\right|+\omega(H[X])\right)>\left|X^{c} \backslash T_{2}\right|$.

We also have $\left|V\left(H /\left(X \cup T_{2}\right)\right)\right|=|V(H / T)|-\left|V\left(H[X] / T_{1}\right)\right|+\omega(H[X])$. By (5),

$$
\eta(H)\left(\left|V\left(H /\left(X \cup T_{2}\right)\right)\right|-\omega(H)\right)>\left|X^{c} \backslash T_{2}\right| .
$$

Since the inequality (6) is strict, $\left|V\left(H /\left(X \cup T_{2}\right)\right)\right|-\omega(H) \neq 0$, and so

$$
\eta(H)>\frac{\left|X^{c} \backslash T_{2}\right|}{\left|V\left(H /\left(X \cup T_{2}\right)\right)\right|-\omega(H)} .
$$

Let $T_{2}^{\prime} \subseteq \mathcal{E}(H / X)$ be the image of $T_{2}$. Since $\left|V\left(H /\left(X \cup T_{2}\right)\right)\right|-\omega(H) \neq$ $0, V\left(H / X\left[T_{2}^{\prime}\right]\right) \neq V(H / X)$. By definition, $\eta(H / X) \leq d\left((H / X) / T_{2}^{\prime}\right)=$ $d\left(H /\left(X \cup T_{2}\right)\right)$, and thus

(8) $\eta(H / X) \leq \frac{\mathcal{E}(H) \backslash\left(X \cup T_{2}\right)}{\left|V\left(H /\left(X \cup T_{2}\right)\right)\right|-\omega(H)} \leq \frac{\left|X^{c} \backslash T_{2}\right|}{\left|V\left(H /\left(X \cup T_{2}\right)\right)\right|-\omega(H)}$.

By (7) and (8), $\eta(H)>\eta(H / X)$, which is impossible by (i). This completes the proof.

(iii) Since $\gamma(H) \geq d(H) \geq l$, by the definition of $\gamma(H)$, there exists a nonempty subset $X \subseteq \mathcal{E}(H)$ such that $\gamma(H)=d(H[X])$. Thus $\gamma(H[X]) \leq$ $\gamma(H)=d(H[X]) \leq \gamma(H[X])$, and we have $\gamma(H[X])=d(H[X]) \geq l$. By Theorem 3.3, $\eta(H[X])=d(H[X])=\gamma(H[X]) \geq l$.

Lemma 3.5. Let $H$ be a nontrivial hypergraph. The following are equivalent.

(i) $H$ is uniformly dense.

(ii) For any nontrivial sub-hypergraph $S, d(S) \leq \eta(H)$.

(iii) For any nontrivial sub-hypergraph $S, \eta(S) \leq \eta(H)$.

Proof. (i) $\Longrightarrow$ (ii). As $H$ is uniformly dense, $d(S) \leq \gamma(H)=\eta(H)$, and so (ii) holds.

(ii) $\Longrightarrow$ (iii). By $(2), \eta(S) \leq d(S) \leq \eta(H)$, and so (iii) holds.

(iii) $\Longrightarrow$ (i). If $H$ is not uniformly dense, then by (2) and (1), for some subset $X \subseteq \mathcal{E}, d(X)=\gamma(H)>\eta(H)$. Let $S=H[X]$. By (1) again, $d(S)=$ $\gamma(S)=\gamma(H)$, and so by Theorem $3.3, \eta(S)=d(S)=\gamma(H)>\eta(H)$, contrary to (iii). This completes the proof. 


\section{Complete families and decompositions}

Throughout this section, unless otherwise stated, sub-hypergraphs of a hypergraph $H$ are all edge induced, and so we adopt the convention to use a subset $S$ of $\mathcal{E}(H)$ to denote both the edge subset as well as the edge induced sub-hypergraph of $H$. In particular, if $S_{1}, S_{2}$ are sub-hypergraphs of $H$, then $S_{1} \cup S_{2}$ denotes the sub-hypergraph of $H$ induced by the edge subset $S_{1} \cup S_{2}$.

Let $k \geq 1$ be an integer and let $\mathcal{T}_{k}$ be the family of all $k$-partitionconnected hypergraphs. Thus $K_{1} \in \mathcal{T}_{k}$ and every hypergraph in $\mathcal{T}_{k}$ is connected. A decomposition theorem that partitions the hyperedges set $\mathcal{E}$ of a hypergraph $H$ according to the different level of partition connectivity, and other related results, will be presented in Theorems 4.4, 4.9, Propositions 4.1 and 4.6 in this section. Connected graph families satisfying properties (C1), (C2) and (C3) stated in Proposition 4.1 are often referred as complete families, as seen in $[3,14,6]$, among others.

Proposition 4.1. For any positive integer $k$, each of the following statements holds.

(C1) $\mathcal{T}_{k} \neq \emptyset$.

(C2) If $E \in \mathcal{E}(H)$ and $H \in \mathcal{T}_{k}$, then $H / E \in \mathcal{T}_{k}$.

(C3) If for some $S \subset \mathcal{E}(H)$, both $S, H / S \in \mathcal{T}_{k}$, then $H \in \mathcal{T}_{k}$.

Proof. Since $K_{1} \in \mathcal{T}_{k}$, (C1) holds.

Let $E=\left\{v_{1}, v_{2}, \ldots, v_{|E|}\right\}$ and $v$ be the vertex of $H / E$ onto which $E$ is contracted. Let $\pi=\left\{V_{1}, V_{2}, \ldots, V_{|\pi|}\right\}$ denote a partition of $V(H / E)$. Without loss of generality, we assume that $v \in V_{1}$. Define $V_{1}^{\prime}=\left(V_{1} \backslash\{v\}\right) \cup$ $\left\{v_{1}, v_{2}, \ldots, v_{|E|}\right\}$. Then $\pi^{\prime}=\left\{V_{1}^{\prime}, V_{2}, \ldots, V_{|\pi|}\right\}$ is a partition of $V(E)$. Since $H \in \mathcal{T}_{k}, e\left(\pi^{\prime}\right) \geq k\left(\left|\pi^{\prime}\right|-1\right)=k(|\pi|-1)$. By the definition of contraction, $e(\pi)=e\left(\pi^{\prime}\right) \geq k(|\pi|-1)$, whence $H / E \in \mathcal{T}_{k}$, and so (C2) follows.

Let $\pi=\left\{V_{1}, V_{2}, \ldots, V_{|\pi|}\right\}$ be a partition of $H$. Without lost of generality, we assume that for some integer $t \geq 1, V_{j} \cap V(S) \neq \emptyset$ for $1 \leq j \leq t$, and $V_{j} \cap$ $V(S)=\emptyset$ for $t+1 \leq j \leq|\pi|$. Then $\pi_{1}=\left\{V_{1} \cap V(S), V_{2} \cap V(S), \ldots, V_{t} \cap V(S)\right\}$ is a partition of $V(S)$. As $S \in \mathcal{T}_{k}, e\left(\pi_{1}\right) \geq k\left(\left|\pi_{1}\right|-1\right)=k(t-1)$. Moreover, let $\pi_{2}=\left\{V_{0}, V_{t+1}, V_{t+2}, \ldots, V_{|\pi|}\right\}$ be a partition of $V(H / S)$. As $H / S \in \mathcal{T}_{k}$, $e\left(\pi_{2}\right) \geq k\left(\left|\pi_{2}\right|-1\right)=k(|\pi|-t)$. It follows that $e(\pi)=e\left(\pi_{1}\right)+e\left(\pi_{2}\right) \geq$ $k(|\pi|-1)$, and so $H \in \mathcal{T}_{k}$. This proves (C3).

Corollary 4.2. If $S_{1}$ and $S_{2}$ are sub-hypergraphs of a hypergraph $H$ such that $S_{1}, S_{2} \in \mathcal{T}_{k}$ and $V\left(S_{1}\right) \cap V\left(S_{2}\right) \neq \emptyset$, then $S_{1} \cup S_{2} \in \mathcal{T}_{k}$.

Proof. Let $H=S_{1} \cup S_{2}$. Since $S_{1} \in \mathcal{T}_{k}$, by Proposition $4.1(\mathrm{C} 2), H / S_{2} \in \mathcal{T}_{k}$. Since $S_{2} \in \mathcal{T}_{k}$, by Proposition 4.1(C3), $H \in \mathcal{T}_{k}$. 
Let $H$ be a nontrivial partition-connected hypergraph. For any positive integer $r$, a nontrivial sub-hypergraph $S$ of $H$ is $\mathcal{T}_{r}$-maximal or $r$ maximal for short, if $S \in \mathcal{T}_{r}$ and if there is no sub-hypergraph $K$ of $H$ such that $K$ contains $S$ properly and such that $K \in \mathcal{T}_{r}$. A $\mathcal{T}_{r}$-maximal sub-hypergraph $S$ of $H$ is an $r$-region if $r=\tau(S)$. Sometimes an $r$-region is also called a region if $r$ is not specified. We define $\bar{\tau}(H)=\max \{r$ : $H$ has a sub-hypergraph as an $r$-region\}.

Lemma 4.3. Let $S$ be a nontrivial connected sub-hypergraph of $H$ and $r$ be a positive integer. If $\tau(S)=r$, then there is always a region $L$ of $H$ with $S \subseteq \mathcal{E}(L)$ and with $\tau(L) \geq r$.

Proof. If $S$ is $r$-maximal, then $L=S$ is an $r$-region of $H$. Otherwise, $H$ has a connected sub-hypergraph $L$ properly containing $S$ with $\tau(L) \geq r$ and such that $L$ is maximal with respect to these properties. Since $H$ is finite, $L$ exists and so $L$ is a desirable region.

Theorem 4.4. Let $H$ be a nontrivial partition-connected hypergraph. Then, (i) There exist a positive integer $m$ and an $m$-tuple $\left(i_{1}, i_{2}, \ldots, i_{m}\right)$ of positive integers with

$$
\tau(H)=i_{1}<i_{2}<\cdots<i_{m}=\bar{\tau}(H)
$$

and a sequence of edge subsets

$$
\mathcal{E}_{m} \subset \cdots \subset \mathcal{E}_{2} \subset \mathcal{E}_{1}=\mathcal{E}(H)
$$

such that each component of the induced sub-hypergraph $H\left[\mathcal{E}_{j}\right]$ is an r-region of $H$ for some $r$ with $r \geq i_{j}$ where $1 \leq j \leq m$, and such that at least one component $S$ in $H\left[\mathcal{E}_{j}\right]$ is an $i_{j}$-region of $H$.

(ii) If $S$ is a sub-hypergraph of $H$ with $\tau(S) \geq i_{j}$, then $\mathcal{E}(S) \subseteq \mathcal{E}_{j}$.

(iii) The integer $m$ and the sequence of edge subsets are uniquely determined by $H$.

Proof. (i) Let $\mathcal{R}(H)$ denote the collection of all regions of $H$. Since $H$ itself is a region of $H, \mathcal{R}(H)$ is not empty. Since $H$ is a finite hypergraph, $|\mathcal{R}(H)|$ is finite. We define $s p(H)=\{\tau(S): S \in \mathcal{R}(H)$ is nontrivial $\}$. Then $|s p(H)|$ is finite and $|s p(H)| \geq 1$. Let $m=|s p(H)|$ and $s p(H)=\left\{i_{1}, i_{2}, \ldots, i_{m}\right\}$ with $i_{1}<i_{2}<\cdots<i_{m}$. Since $H \in \mathcal{R}(H), \tau(H) \geq i_{1}$. If $\tau(H)>i_{1}$, then for some region $S \in \mathcal{R}(H), \tau(S)=i_{1}<\tau(H)$, contrary to the fact that $S$ is a region of $H$. Hence we must have $\tau(H)=i_{1}$.

For each $j \in\{1,2, \ldots, m\}$, we define $\mathcal{E}_{j}=\bigcup_{\tau(S) \geq i_{j}, S \in \mathcal{R}(H)} \mathcal{E}(S)$. As $\mathcal{T}_{i_{1}} \supset \mathcal{T}_{i_{2}} \supset \cdots \supset \mathcal{T}_{i_{m}}$, we have $\mathcal{E}_{1} \supset \mathcal{E}_{2} \supset \cdots \supset \mathcal{E}_{m}$. In particular, $\mathcal{E}_{1}=$ $\cup_{\tau(S) \geq i_{1}} \mathcal{E}(S)=\cup_{\tau(S) \geq \tau(H)} \mathcal{E}(S)=\mathcal{E}(H)$. Claim 1 below completes the proof for (i). 
Claim 1. For any $j \in\{1,2, \ldots, m\}$, each component of $H\left[\mathcal{E}_{j}\right]$ is an $r$-region of $H$ with $r \geq i_{j}$.

Proof of Claim 1. Let $L$ be a nontrivial component of $H\left[\mathcal{E}_{j}\right]$. By the definition of $\mathcal{E}_{j}$, we may assume that there are $s$ regions $L_{1}, L_{2}, \ldots, L_{s}$ such that each $L_{t}$ is an $r_{t}$-region with $r_{t} \geq i_{j}$ for $1 \leq t \leq s$, and such that $L=\cup_{t=1}^{s} L_{t}$. Without loss of generality, we may assume that $r_{1} \leq r_{2} \leq \cdots \leq r_{s}$. If $s \geq 2$, then $L_{1}$ must share a common vertex with some $L_{t}$ with $t \geq 2$ since $L$ is connected. By Corollary 4.2, $L_{1} \cup L_{t} \in \mathcal{T}_{r_{1}}$, contrary to the fact that $L_{1}$ is $r_{1}$-maximal. Hence $s=1$ and $L=L_{1}$. Thus $L$ is an $r_{1}$-region of $H$ with $r_{1} \geq i_{j}$, completing the proof of the claim.

We still need to show that $H\left[\mathcal{E}_{j}\right]$ contains a component as an $i_{j}$-region of $H$. Since $i_{j} \in \operatorname{sp}(H)$, there is an $i_{j}$-region $S$ of $H$, and so $S \subseteq \mathcal{E}_{j}$. The maximality of a region implies that $S$ is a component of $H\left[\mathcal{E}_{j}\right]$.

(ii) follows from Lemma 4.3 and the definition of $\mathcal{E}_{j}$.

(iii) follows from the fact that $\mathcal{R}(H)$ is uniquely determined by $H$.

Theorem 4.4 will be a useful tool to prove our main results in the last two sections. It also has a fractional version to be developed in Theorem 4.9 below.

Lemma 4.5. Let $H$ be a nontrivial connected hypergraph. Then

(i) For some $S \subseteq \mathcal{E}(H), S$ is uniformly dense with $\eta(S)=\gamma(H)$.

(ii) $\bar{\tau}(H)=\lfloor\gamma(H)\rfloor$.

Proof. (i) By (1) and (2), for some $S \subseteq \mathcal{E}(H), S$ is connected and $d(S)=$ $\gamma(H)$. Hence $d(S) \leq \gamma(S) \leq \gamma(S)=d(S)$, and so by Theorem 3.3, $S$ is uniformly dense with $\eta(S)=d(S)=\gamma(H)$. This proves (i).

(ii) By the definition of $\bar{\tau}(H)$, for some region $R$ of $H, \tau(R)=\bar{\tau}(H)$. By (1) and (2),

$$
\bar{\tau}(H)=\tau(R) \leq \eta(R) \leq d(R) \leq \gamma(R) \leq \gamma(H)
$$

Let $k>0$ be an integer with $\gamma(H) \geq k$. By (i), for some $S \subseteq \mathcal{E}(H), S$ is connected and $\eta(S)=\gamma(H) \geq k$. By Lemma 4.3, $H$ has a region $L$ such that $\tau(L) \geq \tau(S) \geq k$. It follows that $\bar{\tau}(H) \geq \tau(L) \geq k$, and so (ii) must hold.

For each rational number $l \geq 0$, we define $\mathcal{S}_{l}=\{H: \eta(H) \geq l\}$.

Proposition 4.6. The hypergraph family $\mathcal{S}_{l}$ has the following properties.

(C1) $\mathcal{S}_{l}$ is nonempty.

(C2) If $H \in \mathcal{S}_{l}$ and $E \in \mathcal{E}(H)$, then $H / E \in \mathcal{S}_{l}$.

(C3) Let $X \subseteq \mathcal{E}(H)$. If $H / X \in \mathcal{S}_{l}$ and $H[X] \in \mathcal{S}_{l}$, then $H \in \mathcal{S}_{l}$. 
Proof. As (C1) and (C2) follow from the fact $K_{1} \in \mathcal{S}_{l}$ and from Lemma 3.4(i), respectively, it suffices to show (C3). Suppose that under the assumption of (C3), we still have $\eta(H)<l$. Then $\eta(H[X]) \geq l>\eta(H)$. By Lemma 3.4(ii), $\eta(H / X)=\eta(H)<l$, contrary to $H / X \in \mathcal{S}_{l}$. Thus $H \in \mathcal{S}_{l}$.

Lemma 4.7. Let $X$ and $X^{\prime}$ be subsets of $\mathcal{E}(H)$ and $l$ be a rational number. If $\eta(X) \geq l$ and $\eta\left(X^{\prime}\right) \geq l$, then $\eta\left(X \cup X^{\prime}\right) \geq l$.

Proof. By Proposition 4.6 (C2), $\left(X \cup X^{\prime}\right) / X=X^{\prime} /\left(X \cap X^{\prime}\right) \in \mathcal{S}_{l}$. As $X^{\prime} \in \mathcal{S}_{l}$, it follows from Proposition 4.6(C3) that $\eta\left(X \cup X^{\prime}\right) \geq l$.

Let $H$ be a nontrivial hypergraph. A subset $S \in \mathcal{E}(H)$ is $\eta$-maximal if for any subset $S^{\prime} \in \mathcal{E}(H)$ with $S \subset S^{\prime}$ properly, we always have $\eta\left(S^{\prime}\right)<\eta(S)$.

Lemma 4.8. Let $S$ be a sub-hypergraph of $H$. Then $H$ has an $\eta$-maximal sub-hypergraph $L$ such that $\mathcal{E}(S) \subseteq \mathcal{E}(L)$ and such that $\eta(S) \leq \eta(L)$.

Proof. Let $l=\eta(S)$ and $\mathcal{F}$ be the collection of all sub-hypergraphs $S^{\prime}$ of $H$ with $\eta\left(S^{\prime}\right) \geq l$. Let $X=\cup_{S^{\prime} \in \mathcal{F}} \mathcal{E}\left(S^{\prime}\right)$ and $L=H[X]$. By Lemma 4.7, $\eta(L) \geq l$. By the definition of $L, L$ is $\eta$-maximal with $\mathcal{E}(S) \subseteq \mathcal{E}(L)$ and $\eta(S) \leq \eta(L)$.

Theorem 4.9. Let $H$ be a nontrivial hypergraph. Then each of the following holds.

(i) There exist a positive integer $m$ and an $m$-tuple $\left(l_{1}, l_{2}, \ldots, l_{m}\right)$ of positive rational numbers with

$$
\eta(H)=l_{1}<l_{2}<\cdots<l_{m}=\gamma(H)
$$

and a sequence of edge subsets

$$
J_{m} \subset \cdots \subset J_{2} \subset J_{1}=\mathcal{E}(H)
$$

such that for each $i$ with $1 \leq i \leq m, J_{i}$ is $\eta$-maximal with $\eta\left(H\left[J_{i}\right]\right)=l_{i}$.

(ii) The integer $m$ and the sequences above are uniquely determined by $H$.

Proof. Let $\mathcal{R}(H)$ denote the collection of all $\eta$-maximal sub-hypergraphs of $H$. Then $H \in \mathcal{R}(H)$ and $|\mathcal{R}(H)|$ are finite. Let $s p_{\eta}(H)=\{\eta(S): S \in$ $\mathcal{R}(H)\}, m=\left|s p_{\eta}(H)\right|$ and $s p_{\eta}(H)=\left\{l_{1}, l_{2}, \ldots, l_{m}\right\}$ such that $l_{1}<l_{2}<$ $\cdots<l_{m}$.

Since $H \in \mathcal{R}(H), \eta(H) \geq l_{1}$. If for some $K \in \mathcal{R}(H)$, with $\eta(K)=l_{1}<$ $\eta(H)$, then $K$ is not $\eta$-maximal. Therefore, $\eta(H)=l_{1}$. By Lemma 4.5(i), $\gamma(H) \leq l_{m}$. If for some $K \in \mathcal{R}(H)$, with $\eta(K)=l_{m}>\gamma(H)$, then by (2), $d(K) \geq \eta(K)>\gamma(H)$, contrary to (1). Therefore, $\gamma(H)=l_{m}$. 
Fix an $i$ with $1 \leq i \leq m$, by the definition of $l_{i}$, for some $S \in \mathcal{R}(H)$, $\eta(S)=l_{i}$. Define $J_{i}$ to be the set of all hyperedges of $H$ which are in some $S \in \mathcal{R}(H)$ with $\eta(S)=l_{i}$. Then by Proposition $4.6(\mathrm{C} 3), J_{m} \subset \cdots \subset J_{2} \subset$ $J_{1}=\mathcal{E}(H)$. This proves (i).

(ii) follows from the fact that $\mathcal{R}(H)$ is uniquely determined by $H$.

The $m$-tuple $\left(l_{1}, l_{2}, \ldots, l_{m}\right)$ in (9) and the sequence $J_{1}, J_{2}, \ldots, J_{m}$ in (10) are referred as the $\eta$-spectrum and the $\eta$-decomposition of $H$, respectively.

Corollary 4.10. Let $H$ be a nontrivial hypergraph with $\eta$-spectrum and $\eta$ decomposition described in Theorem 4.9 with $m>1$. Then $H / J_{2}$ is uniformly dense with $\eta\left(H / J_{2}\right)=\gamma\left(H / J_{2}\right)=\eta(H)$.

Proof. Since $m>1, \eta\left(H\left[J_{2}\right]\right)=l_{2}>l_{1}=\eta(H)$. By Lemma 3.4(ii), $\eta\left(H / J_{2}\right)=\eta(H)=l_{1}$. It remains to show that $\gamma\left(H / J_{2}\right)=\eta\left(H / J_{2}\right)$.

If not, then by Lemma 4.5(i) and by (1) and (2), for some $J^{\prime} \subset \mathcal{E}\left(H / J_{2}\right)$, $\eta\left(H / J_{2}\left[J^{\prime}\right]\right)=d_{H / J_{2}}\left(J^{\prime}\right)=\gamma\left(H / J_{2}\right)>\eta\left(H / J_{2}\right)=l_{1}$. Let $J^{\prime \prime} \subseteq \mathcal{E}(H)$ be a preimage of $J^{\prime}$. Then $J^{\prime \prime} \cap J_{2}=\emptyset$ and, since $J_{2}$ is $\eta$-maximal, $\eta\left(J^{\prime \prime} \cup J_{2}\right)<$ $\eta\left(J_{2}\right)=l_{2}$. By Lemma 3.4(ii), $\eta\left(J^{\prime \prime} \cup J_{2}\right)=\eta\left(\left(J^{\prime \prime} \cup J_{2}\right) / J_{2}\right)=\eta\left(H / J_{2}\left[J^{\prime}\right]\right)>$ $l_{1}$. By Lemma $4.8, H$ has an $\eta$-maximal sub-hypergraph $L$ with $\eta(L) \geq$ $\eta\left(J^{\prime \prime} \cup J_{2}\right)$ with $J^{\prime \prime} \cup J_{2} \subseteq L$. If $\eta(L) \geq l_{2}$, then $L \subseteq J_{2}$, contrary to $J^{\prime \prime} \cap J_{2}=\emptyset$. Hence $l_{2}>\eta(L) \geq \eta\left(J^{\prime \prime} \cup J_{2}\right)>l_{1}$, and so the $\eta$-spectrum of $H$ should include $\eta(L)$, contrary to the uniqueness of the $\eta$-spectrum of $H$.

Corollary 4.11. Let $H$ be a hypergraph with $\eta$-spectrum (9). Then $H$ is uniformly dense if and only if $m=1$.

\section{Augmenting partition connectivity of a hypergraph}

Throughout this section, $k>0$ denotes an integer, and $H$ denotes a hypergraph. If $X$ is a collection of (not necessarily distinct) subsets of $V(H)$ and $X \cap \mathcal{E}(H)=\emptyset$, then we use $H+X$ to denote the hypergraph $(V(H), \mathcal{E} \cup X)$. Define $f(H, k)$ to be the minimum number of hyperedges that must be added to $H$ so that the resulting hypergraph is $k$-partition-connected. By Theorem 2.3, it suffices to investigate the minimum number of hyperedges that must be added to $H$ so that the resulting hypergraph has $k$ edge-disjoint spanning hypertrees. In this section, we determine the value of $f(H, k)$ together with a min-max formula (Theorems 5.4 and 5.8). Matroid arguments will be used in some of the proofs, and we refer to [20] for undefined terms for matroid theory.

Lemma 5.1. Every hyperforest in a partition-connected hypergraph is a spanning sub-hypergraph of a hypertree. 
Proof. Lorea [17] proved that all hyperforests of a hypergraph $H$ form the family of independent sets of a matroid $M_{H}$, called the circuit matroid of $H$, on $\mathcal{E}(H)$. Frank, Király and Kriesell [10] proved that, if $H$ is partitionconnected, then any spanning hypertree of $H$ is a base of $M_{H}$. It follows that any hyperforest in a partition-connected hypergraph can be augmented to a hypertree.

Lemma 5.2. Suppose that $\tau(H)<k$. If $\gamma(H) \leq k$, then there exists an edge set $X$ with $|X|=k(|V(H)|-1)-|\mathcal{E}(H)|$ such that $H+X$ is the union of $k$ edge-disjoint spanning hypertrees.

Proof. Since $\gamma(H) \leq k$, by Theorem 2.4 or Proposition 3.1, there exist edgedisjoint spanning hyperforests $F_{1}, F_{2}, \ldots, F_{k}$ such that $\mathcal{E}(H)=\cup_{i=1}^{k} \mathcal{E}\left(F_{i}\right)$. By Lemma 5.1, for each $i$ with $1 \leq i \leq k$, each $F_{i}$ can be augmented to a hypertree by adding a set $X_{i}^{\prime}$ of $\left|V\left(F_{i}\right)\right|-1-\left|\mathcal{E}\left(F_{i}\right)\right|$ hyperedges. For each $i$ with $1 \leq i \leq k$, let $X_{i}$ be a set of new hyperedges duplicating the edges in $X_{i}^{\prime}$, and let $X=\cup_{i=1}^{k} X_{i}$. Then $H+X$ is the union of $k$ edge-disjoint spanning hypertrees and $|X|=\sum_{i=1}^{k}\left(\left|V\left(F_{i}\right)\right|-1-\left|\mathcal{E}\left(F_{i}\right)\right|\right)=k(|V(H)|-$ $1)-|\mathcal{E}(H)|$.

Lemma 5.3. Let $H$ be a hypergraph and let $W \subseteq \mathcal{E}(H)$ such that every component of $W$ is in $\mathcal{T}_{k}$. If for a set $X^{\prime}$ of hyperedges not in $\mathcal{E}(H / W)$, $H / W+X^{\prime} \in \mathcal{T}_{k}$, then for some set $X$ of hyperedges not in $\mathcal{E}(H), H+X \in \mathcal{T}_{k}$ and $|X|=\left|X^{\prime}\right|$.

Proof. Suppose that $H[W]$ has $c$ components $H_{1}, H_{2}, \ldots, H_{c}$ and let $v_{1}, v_{2}, \ldots, v_{c}$ be the vertices in $H / W$ onto which $H_{1}, H_{2}, \ldots, H_{c}$ are contracted, respectively. We will construct an edge set $X$ from $X^{\prime}$ as follows: Label $X^{\prime}=\left\{E_{1}^{\prime}, E_{2}^{\prime}, \ldots, E_{s}^{\prime}\right\}$, where $s=\left|X^{\prime}\right|$. For each $i$ with $1 \leq i \leq s$, we have the following.

(a) If $E_{i}^{\prime} \cap\left\{v_{1}, v_{2}, \ldots, v_{c}\right\}=\emptyset$, then $E_{i}=E_{i}^{\prime} \in X$.

(b) If $E_{i}^{\prime} \cap\left\{v_{1}, v_{2}, \ldots, v_{c}\right\}=\left\{v_{i_{1}}, v_{i_{2}}, \ldots, v_{i_{t}}\right\}$ for some $1 \leq t \leq c$, then choose $u_{j} \in V\left(H_{i_{j}}\right)$ for each $j$ with $1 \leq j \leq t$, and define $E_{i}=\left(E_{i}^{\prime} \backslash\left\{v_{1}, v_{2}, \ldots, v_{t}\right\}\right) \cup$ $\left\{u_{1}, u_{2}, \ldots, u_{t}\right\}$.

Therefore, $|X|=\left|X^{\prime}\right|$. By the definition of contraction, $H / W+X^{\prime} \cong$ $(H+X) / W$. Since $H_{i} \in \mathcal{T}_{k}$, and since $(H+X) / W \cong H / W+X^{\prime} \in \mathcal{T}_{k}$, by Proposition 4.1, $H+X \in \mathcal{T}_{k}$.

Let $H$ be a partition-connected hypergraph and $i_{j}, \mathcal{E}_{j}$ be defined in Theorem 4.4 for $j=1,2, \ldots, m$. Let $k$ be a positive integer. If $k \leq i_{m}$, we define $i(k)=\min \left\{i_{j}: i_{j} \geq k\right\}$. If $k>i_{m}$, we define $i(k)=\infty$ and $\mathcal{E}_{\infty}=\emptyset$. Let 
$c_{k}(H)$ be the number of components of $H\left[\mathcal{E}_{i(k)}\right]$ and $w_{k}(H)=\left|V\left(H\left[\mathcal{E}_{i(k)}\right]\right)\right|$. Note that $c_{k}(H)=w_{k}(H)=0$ if $i(k)=\infty$.

Theorem 5.4. Let $H$ be a partition-connected hypergraph with $\tau(H)<k$. Then $f(H, k)=k\left(|V(H)|-w_{k}(H)+c_{k}(H)-1\right)-\left(|\mathcal{E}(H)|-\left|\mathcal{E}_{i(k)}\right|\right)$.

Proof. If $\gamma(H)<k$, then by Lemma 4.5, $i_{m}=\bar{\tau}(H) \leq \gamma(H)<k$. Then $i(k)=\infty$, and we have $c_{k}(H)=w_{k}(H)=0$. Then the theorem follows from Lemma 5.2. Hence we may assume that $\gamma(H) \geq k$. In this case, we define $H^{\prime}=H / \mathcal{E}_{i(k)}$, and so both $\left|\mathcal{E}\left(H^{\prime}\right)\right|=|\mathcal{E}(H)|-\left|\mathcal{E}_{i(k)}\right|$ and $\left|V\left(H^{\prime}\right)\right|=|V(H)|-w_{k}(H)+c_{k}(H)$.

Claim 2. $\gamma\left(H^{\prime}\right) \leq k$.

Proof of Claim 2. By contradiction, we assume that $\gamma\left(H^{\prime}\right)>k$.

By Lemma 4.5, $H^{\prime}$ has an $r$-region $L^{\prime}$ with $r \geq k$. Suppose that $H\left[\mathcal{E}_{i(k)}\right]$ has $c$ components $H_{1}, H_{2}, \ldots, H_{c}$ and let $v_{1}, v_{2}, \ldots, v_{c}$ be the vertices in $H / \mathcal{E}_{i(k)}$ that the components $H_{1}, H_{2}, \ldots, H_{c}$ are contracted, respectively. By Theorem 4.4, $\tau\left(H_{i}\right) \geq k$ for $i=1,2, \ldots, c$. If $V\left(L^{\prime}\right) \cap\left\{v_{1}, v_{2}, \ldots, v_{c}\right\}=$ $\emptyset$, then $L^{\prime}$ is a sub-hypergraph of $H$ with $\tau\left(L^{\prime}\right) \geq k$. By Theorem 4.4, $\mathcal{E}\left(L^{\prime}\right) \subseteq \mathcal{E}_{i(k)}$, contrary to the fact that $L^{\prime}$ is a sub-hypergraph of $H / \mathcal{E}_{i(k)}$. If $V\left(L^{\prime}\right) \cap\left\{v_{1}, v_{2}, \ldots, v_{c}\right\} \neq \emptyset$, then, without loss of generality, we may assume that $V\left(L^{\prime}\right) \cap\left\{v_{1}, v_{2}, \ldots, v_{c}\right\}=\left\{v_{1}, v_{2}, \ldots, v_{t}\right\}$ for some $t \leq c$. Let $\mathcal{E}_{\text {pre }}$ be the set of preimages of edges of $\mathcal{E}\left(L^{\prime}\right)$ in $H$, and let $L=H\left[\cup_{i=1}^{t} \mathcal{E}\left(H_{i}\right) \cup \mathcal{E}_{\text {pre }}\right]$. Then $L^{\prime}=L / \cup_{i=1}^{t} \mathcal{E}\left(H_{i}\right)$. Since $L^{\prime} \in \mathcal{T}_{k}$ and each component of $H\left[\cup_{i=1}^{t} \mathcal{E}\left(H_{i}\right)\right]$ is in $\mathcal{T}_{k}$, by Proposition $4.1, L \in \mathcal{T}_{k}$. By Theorem 4.4, $\mathcal{E}(L) \subseteq \mathcal{E}_{i(k)}$, contrary to the fact that $L^{\prime}$ is a sub-hypergraph of $H^{\prime}$. This proves the claim.

By Claim 2 and Lemma 5.2, there exists an edge set $X^{\prime}$ disjoint from $\mathcal{E}(H)$ with $\left|X^{\prime}\right|=k\left(\left|V\left(H^{\prime}\right)\right|-1\right)-\left|\mathcal{E}\left(H^{\prime}\right)\right|$ such that $H^{\prime}+X^{\prime}$ is the union of $k$ edge-disjoint spanning hypertrees. This is the minimum number of hyperedges that must be added to $H^{\prime}$ in order to have $k$ edge-disjoint spanning hypertrees.

By Lemma 5.3 with $W=\mathcal{E}_{i(k)}$, for some edge subset $X$ disjoint from $\mathcal{E}(H)$, with $|X|=\left|X^{\prime}\right|$, such that $H+X \in \mathcal{T}_{k}$. Thus $f(H, k)=k\left(\left|V\left(H^{\prime}\right)\right|-\right.$ $1)-\left|\mathcal{E}\left(H^{\prime}\right)\right|=k\left(|V(H)|-w_{k}(H)+c_{k}(H)-1\right)-\left(|\mathcal{E}(H)|-\left|\mathcal{E}_{i(k)}\right|\right)$.

In the rest of this section, we present a related min-max formula for $f(H, k)$ (Theorem 5.8). For any subset $X \subseteq \mathcal{E}(H)$, define

$$
f_{k}(H, X)=k(\omega(H-X)-1)-|X| \text { and } F_{k}(H)=\max _{X \subseteq \mathcal{E}(H)}\left\{f_{k}(H, X)\right\} .
$$

Note that $F_{k}(H) \geq f_{k}(H, \emptyset)=0$. 
Lemma 5.5. If $X \subseteq \mathcal{E}(H)$ is a subset with $f_{k}(H, X)=F_{k}(H)$ and if $C$ is a component of $H-X$, then each of the following holds.

(i) For any subset $X_{C}$ of $\mathcal{E}(C), f_{k}\left(H, X \cup X_{C}\right)=f_{k}(H, X)+f_{k}\left(C, X_{C}\right)$.

(ii) $F_{k}(C)=0$.

(iii) $\tau(C) \geq k$ (and so $C \in \mathcal{T}_{k}$ ).

Proof. (i) $f_{k}\left(H, X \cup X_{C}\right)=k\left(\omega\left(H-\left(X \cup X_{C}\right)\right)-1\right)-\left|X \cup X_{C}\right|=k(\omega(H-$ $\left.X)-1+\omega\left(C-X_{C}\right)-1\right)-|X|-\left|X_{C}\right|=k(\omega(H-X)-1)-|X|+k(\omega(C-$ $\left.\left.X_{C}\right)-1\right)-\left|X_{C}\right|=f_{k}(H, X)+f_{k}\left(C, X_{C}\right)$.

(ii) By (i), for any $X_{C} \subseteq \mathcal{E}(C), f_{k}\left(C, X_{C}\right)=f_{k}\left(H, X \cup X_{C}\right)-f_{k}(H, X)=$ $f_{k}\left(H, X \cup X_{C}\right)-F_{k}(H) \leq 0$. Thus $F_{k}(C)=0$.

(iii) By (ii), for any $X_{C} \subseteq \mathcal{E}(C), f_{k}\left(C, X_{C}\right) \leq 0$. In particular, for any $X_{C} \subseteq \mathcal{E}(C)$ with $\omega\left(C-X_{C}\right)>1, k\left(\omega\left(C-X_{C}\right)-1\right)-\left|X_{C}\right| \leq 0$. Thus $\frac{\left|\bar{X}_{C}\right|}{\omega\left(C-X_{C}\right)-1} \geq k$. By Theorem 2.3, $\tau(C) \geq k$.

Lemma 5.6. If $H$ is connected and $F_{k}(H)=f_{k}(H, \mathcal{E}(H))$, then $\gamma(H) \leq k$.

Proof. Let $S$ be an induced sub-hypergraph of $H$. By the definition of $\gamma(H)$, it suffices to show that $|\mathcal{E}(S)| \leq k(|V(S)|-\omega(S))$. By definition, $F_{k}(H)=$ $f_{k}(H, \mathcal{E}(H))=k(|V(H)|-1)-|\mathcal{E}(H)|$. Let $X=\mathcal{E}(H) \backslash \mathcal{E}(S)$. Then the components of $H-X$ is the components of $S$ and $|V(H)|-|V(S)|$ isolated vertices. Thus $f_{k}(H, X)=k(\omega(H-X)-1)-|X|=k(\omega(S)+|V(H)|-$ $|V(S)|-1)-(|\mathcal{E}(H)|-|\mathcal{E}(S)|)=k(|V(H)|-1)-|\mathcal{E}(H)|+k(\omega(S)-|V(S)|)+$ $|\mathcal{E}(S)|=F_{k}(H)-k(|V(S)|-\omega(S))+|\mathcal{E}(S)|$. Since $f_{k}(H, X) \leq F_{k}(H)$, we have $F_{k}(H)-k(|V(S)|-\omega(S))+|\mathcal{E}(S)| \leq F_{k}(H)$, that is, $|\mathcal{E}(S)| \leq$ $k(|V(S)|-\omega(S))$, completing the proof.

Lemma 5.7. Let $H$ be a hypergraph and $X$ be a subset of $\mathcal{E}(H)$ such that $f_{k}(H, X)=F_{k}(H)$. Let $H_{0}=H /(\mathcal{E}(H) \backslash X)$ and $X_{0} \subseteq \mathcal{E}\left(H_{0}\right)$ be the image of $X$. Then $f_{k}\left(H_{0}, X_{0}\right)=F_{k}\left(H_{0}\right)=F_{k}(H)$.

Proof. First noticing that $\omega(H-X)=\omega\left(H_{0}-X_{0}\right)$ and $\left|X_{0}\right| \leq|X|$ (this is because the images of some hyperedges might be loops and will be removed), by the definition of $f_{k}(H, X)$, we have $f_{k}(H, X) \leq f_{k}\left(H_{0}, X\right)$. Thus $F_{k}\left(H_{0}\right) \geq f_{k}\left(H_{0}, X_{0}\right) \geq f_{k}(H, X)=F_{k}(H)$. On the other hand, we may choose $X_{0}^{\prime} \subseteq X_{0}$ such that $F_{k}\left(H_{0}\right)=f_{k}\left(H_{0}, X_{0}^{\prime}\right)$. Let $X^{\prime} \subseteq \mathcal{E}(H)$ be a set of preimages of hyperedges of $X_{0}^{\prime}$. Then $\left|X^{\prime}\right|=\left|X_{0}^{\prime}\right|$. Since $\omega\left(H-X^{\prime}\right)=$ $\omega\left(H_{0}-X_{0}^{\prime}\right)$, we have $f_{k}\left(H, X^{\prime}\right)=f_{k}\left(H_{0}, X_{0}^{\prime}\right)$, and thus $F_{k}(H) \geq f_{k}\left(H, X^{\prime}\right)=$ $f_{k}\left(H_{0}, X_{0}^{\prime}\right)=F_{k}\left(H_{0}\right)$. It follows that $f_{k}\left(H_{0}, X_{0}\right)=F_{k}\left(H_{0}\right)=F_{k}(H)$.

Theorem 5.8. Let $H$ be a connected hypergraph. Then $f(H, k)=F_{k}(H)$. 
Proof. Let $X$ be a subset of $\mathcal{E}(H)$ such that $f_{k}(H, X)=F_{k}(H)$. Let $H_{0}=H /(\mathcal{E}(H) \backslash X)$ and $X_{0} \subseteq \mathcal{E}\left(H_{0}\right)$ be the image of $X$. By Lemma 5.7, $f_{k}\left(H_{0}, X\right)=F_{k}\left(H_{0}\right)=F_{k}(H)$. By Lemma 5.6, $\gamma\left(H_{0}\right) \leq k$. Thus, by Lemma 5.2, $f\left(H_{0}, k\right)=k\left(\left|V\left(H_{0}\right)\right|-1\right)-\left|\mathcal{E}\left(H_{0}\right)\right|=f_{k}\left(H_{0}, X_{0}\right)=F_{k}\left(H_{0}\right)=$ $F_{k}(H)$.

Let $W=\mathcal{E}(H) \backslash X$. By Lemma 5.5, each component of $W$ is in $\mathcal{T}_{k}$. Let $Y_{0}$ be the edge set with $\left|Y_{0}\right|=f\left(H_{0}, k\right)$ such that $\tau\left(H_{0}+Y_{0}\right) \geq k$. By Lemma 5.3, there exists a set $Y$ of hyperedges not in $\mathcal{E}(H)$ such that $H+Y \in \mathcal{T}_{k}$ with $|Y|=\left|Y_{0}\right|$. Thus $f(H, k) \leq f\left(H_{0}, k\right)=F_{k}(H)$.

To prove $f(H, k) \geq F_{k}(H)$, we assume that $Z$ is a set of hyperedges such that $\tau(H+Z) \geq k$ and $|Z|=f(H, k)$. Let $Z^{\prime} \subseteq \mathcal{E}((H+Z) / W)$ be the image of $Z$. Then $\left|Z^{\prime}\right| \leq|Z|$ and $(H+Z) / W=H / W+Z^{\prime}=H_{0}+Z^{\prime}$. Since $\tau(H+Z) \geq k$, by Proposition 4.1, $\tau\left(H_{0}+Z^{\prime}\right) \geq k$. Thus $F_{k}(H)=$ $f\left(H_{0}, k\right) \leq\left|Z^{\prime}\right| \leq|Z|=f(H, k)$, completing the proof.

\section{Preserving partition connectivity of a hypergraph}

For a positive integer $k$ and a hypergraph $H$ with $\tau(H) \geq k$, we define $\mathcal{E}_{k}(H)=\{E \in \mathcal{E}(H): \tau(H-E) \geq k\}$. The main goal of this section is to determine the set $\mathcal{E}_{k}(H)$ for a $k$-partition-connected hypergraph $H$, as stated in Theorem 6.2 below. We start with a lemma.

Lemma 6.1. Let $H$ be a hypergraph. If there exists $X \subseteq \mathcal{E}(H)$ such that (a) $\tau(H / X) \geq k$ and $\tau(H[X]) \geq k$, and

(b) $\mathcal{E}_{k}(H[X])=\mathcal{E}(H[X])$ and $\mathcal{E}_{k}(H / X)=\mathcal{E}(H / X)$, then $\mathcal{E}_{k}(H)=\mathcal{E}(H)$.

Proof. For any $E \in \mathcal{E}(H)$, if $E \in X$, then by $\mathcal{E}_{k}(H[X])=\mathcal{E}(H[X])$, we have $\tau(H[X]-E) \geq k$. We also have $\tau((H-E) /(X-E))=\tau(H / X) \geq k$. By Proposition 4.1(C3), $\tau(H-E) \geq k$. If $E \notin X$, then let $E^{\prime} \in \mathcal{E}(H / X)$ be the image of $E$. Since $\mathcal{E}_{k}(H / X)=\mathcal{E}(H / X), \tau\left(H / X-E^{\prime}\right) \geq k$. Thus $\tau((H-$ $E) / X)=\tau\left(H / X-E^{\prime}\right) \geq k$. We also have $\tau((H-E)[X])=\tau(H[X]) \geq k$. By Proposition 4.1(C3), $\tau(H-E) \geq k$. Hence $\mathcal{E}_{k}(H)=\mathcal{E}(H)$.

Theorem 6.2. Let $k$ be a positive integer and $H$ be a hypergraph with $\tau(H) \geq k$ and $\eta$-decomposition (10). Then each of the following holds.

(i) $\mathcal{E}_{k}(H)=\emptyset$ if and only if $d(H)=k$.

(ii) $\mathcal{E}_{k}(H)=\mathcal{E}(H)$ if and only if $\eta(H)>k$.

(iii) If $\eta(H)=k$, then $\mathcal{E}_{k}(H)=J_{2}$.

Proof. (i) Since $\tau(H) \geq k, d(H)=k$ if and only if $|\mathcal{E}(H)|=k(|V(H)|-1)$, if and only if $H$ is a union of $k$ edge-disjoint spanning hypertrees, and if and only if $\mathcal{E}_{k}(H)=\emptyset$. 
(ii) By Proposition 3.1, $\eta(H) \geq \tau(H) \geq k$. We argue by contradiction to prove the necessity. Suppose that $\eta(H)=k$. Let $\left(l_{1}, l_{2}, \ldots, l_{m}\right)$ and the sequence $J_{1}, J_{2}, \ldots, J_{m}$ be the $\eta$-spectrum and the $\eta$-decomposition of $H$. By Corollary 4.10, $d\left(H / J_{2}\right)=\eta\left(H / J_{2}\right)=\gamma\left(H / J_{2}\right)=\eta(H)=k$. By (i), for any $E^{\prime} \in \mathcal{E}\left(H / J_{2}\right)$ and its preimage $E \in \mathcal{E}(H), \tau\left((H-E) / J_{2}\right)=\tau\left(H / J_{2}-E^{\prime}\right)<$ $k$. By Proposition $4.1(\mathrm{C} 2), \tau(H-E)<k$, contrary to $\mathcal{E}_{k}(H)=\mathcal{E}(H)$. This proves the necessity. To prove the sufficiency, let $H$ be a hypergraph with

$$
\eta(H)>k \text { and } \mathcal{E}_{k}(H) \neq \mathcal{E}(H) \text { such that } V(H) \text { is minimized. }
$$

Since $\mathcal{E}_{k}(H) \neq \mathcal{E}(H)$, there exists $E_{0} \in \mathcal{E}(H)$ such that

$$
\tau\left(H-E_{0}\right) \leq k-1 .
$$

Claim 3. For any nontrivial sub-hypergraph $S$ of $H$ with $|V(S)|<|V(H)|$, $\eta(S) \leq k$.

Proof of Claim 3. Suppose not and we have $\eta(S)>k$. By $(11), \mathcal{E}_{k}(S)=$ $\mathcal{E}(S)$. By Lemma 3.4(i), $\eta(H / S) \geq \eta(S)>k$, and so by $(11), \mathcal{E}_{k}(H / S)=$ $\mathcal{E}(H / S)$. It follows from Lemma 6.1 that $\mathcal{E}_{k}(H)=\mathcal{E}(H)$, contrary to (11). This proves Claim 3.

By Claim 3, for any $S \subseteq \mathcal{E}(H), \eta(S) \leq k<\eta(H)$. By Lemma 3.5(ii), $H$ is uniformly dense. Then $d(H)=\eta(H)>k$, and so $|\mathcal{E}(H)| \geq k(|V(H)|-1)+1$. We have $d\left(H-E_{0}\right)=\frac{\left|\mathcal{E}\left(H-E_{0}\right)\right|}{\left|V\left(H-E_{0}\right)\right|-\omega\left(H-E_{0}\right)} \geq \frac{|\mathcal{E}(H)|-1}{|V(H)|-1} \geq k$. By Lemma 3.4(iii), there exists a nonempty subset $X \subseteq \mathcal{E}\left(H-E_{0}\right)$ such that $\eta\left(\left(H-E_{0}\right)[X]\right) \geq k$. Thus $\tau\left(\left(H-E_{0}\right)[X]\right)=\left\lfloor\eta\left(\left(H-E_{0}\right)[X]\right)\right\rfloor \geq k$.

By Lemma 3.4(i), $\eta(H / X) \geq \eta(H)>k$. Let $E_{0}^{\prime} \in \mathcal{E}(H / X)$. By (11), $H$ is a minimal counterexample, and so $\tau\left(H / X-E_{0}^{\prime}\right) \geq k$. Thus $\tau\left(\left(H-E_{0}\right) / X\right)=$ $\tau\left(H / X-E_{0}^{\prime}\right) \geq k$. As $\tau\left(\left(H-E_{0}\right)[X]\right) \geq k$, by Proposition 4.1(C3), $\tau(H-$ $\left.E_{0}\right) \geq k$, contrary to (12). This completes the proof of the sufficiency.

(iii) Suppose that $\eta(H)=k$. If $d(H)=k$, then by (i), $\mathcal{E}_{k}(H)=\emptyset$. On the other hand, by Theorem 3.3, $H$ is uniformly dense. By Corollary 4.11, $m=1$ and so $J_{2}=\emptyset$. Thus $\mathcal{E}_{k}(H)=J_{2}$ if $d(H)=k$. Now assume that $d(H)>k$. Then $H$ is not uniformly dense, and so by Corollary 4.11, $m>1$. Suppose that $H$ has $\eta$-spectrum (9) and $\eta$-decomposition (10). By Theorem 4.9, $\eta\left(H\left[J_{2}\right]\right)=l_{2}>l_{1}=\eta(H)=k$. It follows from (ii) that $\mathcal{E}_{k}\left(H\left[J_{2}\right]\right)=J_{2}$. By Corollary $4.10, H / J_{2}$ is uniformly dense with $\eta\left(H / J_{2}\right)=d\left(H / J_{2}\right)=k$, and so by (i), $\mathcal{E}_{k}\left(H / J_{2}\right)=\emptyset$. Then for any hyperedge $E \in J_{2}, \tau((H-$ $\left.E)\left[J_{2}-E\right]\right)=\tau\left(H\left[J_{2}\right]-E\right)=k$ and $\tau\left((H-E) /\left(J_{2}-E\right)\right)=\tau\left(H / J_{2}\right)=k$. By Proposition 4.1(C3), $\tau(H-E)=k$. Thus $J_{2} \subseteq \mathcal{E}_{k}(H)$. To complete 
the proof, we still need to show that $\mathcal{E}_{k}(H) \subseteq J_{2}$. It suffices to prove that for any $E \in \mathcal{E}(H) \backslash J_{2}, \tau(H-E)<k$. If not, we have $\tau(H-E)=k$ and let $E^{\prime} \in \mathcal{E}\left(H / J_{2}\right)$ be the image of $E$, and by Proposition 4.1(C2), $\tau\left(H / J_{2}-E^{\prime}\right)=\tau\left((H-E) / J_{2}\right)=k$, contrary to $\mathcal{E}_{k}\left(H / J_{2}\right)=\emptyset$. Hence $\mathcal{E}_{k}(H)=J_{2}$.

\section{References}

[1] Berge, C. (1989). Hypergraphs: Combinatorics of finite sets, NorthHolland. MR1013569

[2] Bondy, J. A. and Murty, U. S. R. (2008). Graph Theory, Springer, New York. MR2368647

[3] Catlin, P. A. (1996). The reduction of graph families closed under contraction, Discrete Math. 160 67-80. MR1417561

[4] Catlin, P. A., Grossman, J. W. and Hobbs, A. M. (1988). Graphs with uniform density, Congr. Numer. 65 281-286. MR0992879

[5] Catlin, P. A., Grossman, J. W., Hobbs, A. M. and Lai, H.-J. (1992). Fractional arboricity, strength, and principal partitions in graphs and matroids, Discrete Applied Math. 40 285-302. MR1197004

[6] Catlin, P. A., Hobbs, A. M. and Lai, H.-J. (2001). Graph families operations, Discrete Math. 230 71-97. MR1812343

[7] Edmonds, J. (1965). Lehman's switching game and a theorem of Tutte and Nash-Williams, J. Res. Nat. Bur. Standards Sect. B 69 73-77. MR0190026

[8] Frank, A. (1992). Augmenting graphs to meet edge-connectivity requirements, SIAM J. Discrete Math. 5 22-53. MR1146895

[9] Frank, A. and Király, T. (2003). Combined connectivity augmentation and orientation problems, Discrete Applied Math. 131 401-419. MR2021109

[10] Frank, A., Király, T. and Kriesell, M. (2003). On decomposing a hypergraph into $k$ connected sub-hypergraphs, Discrete Applied Math. 131 373-383. MR2021107

[11] Haas, R. (2002). Characterizations of arboricity of graphs, Ars Combin. 63 129-137. MR1898221

[12] Jordán, T. (2012). Minimax theorems in graph connectivity augmentation, EGRES Tech. Rep. 2012-2014.

[13] Király, T. and Makai, M. (2002). A note on hypergraph connectivity augmentation, EGRES Tech. Rep. 2002-2011.

[14] Lai, H.-J. and Lai, H. (1992). Duality in graph families, Discrete Math. 110 165-177. MR1197450 
[15] Li, P., Lai, H.-J. and Liang, Y. (2012). Characterization of removable elements with respect to having $k$ disjoint bases in a matroid, Discrete Applied Math. 160 2445-2451. MR2957953

[16] Liu, D., Lai, H.-J. and Chen, Z.-H. (2009). Reinforcing the number of disjoint spanning trees, Ars Combin. 93 113-127. MR2566744

[17] Lorea, M. (1975). Hypergraphes et matroides, Cahiers Centre Etudes Rech. Oper. 17 289-291. MR0404022

[18] Nash-Williams, C. St. J. A. (1961). Edge-disjoint spanning trees of finite graphs, J. London Math. Soc. 36 445-450. MR0133253

[19] Nash-Williams, C. St. J. A. (1964). Decompositions of finite graphs into forests, J. London Math. Soc. 39 12. MR0161333

[20] Oxley, J. G. (2011). Matroid Theory, $2^{\text {nd }}$ edition, Oxford University Press, New York. MR2849819

[21] Szigeti, Z. (2009). Edge-connectivity augmentations of graphs and hypergraphs, In: W. Cook, L. Lovász and J. Vygen (eds.), Research trends in combinatorial optimization, Springer, Berlin, 483-521. MR2513329

[22] Tutte, W. T. (1961). On the problem of decomposing a graph into $n$ connected factors, J. London Math. Soc. 36 221-230. MR0140438

[23] Watanabe, T. and Nakamura, A. (1987). Edge-connectivity augmentation problems, J. Comput. Syst. Sci. 35 96-144. MR0903181

XiaOfeng Gu

Department of Mathematics and Computer Science

UNIVERSITY OF WISCONSIN-SUPERIOR

SUPERIOR, WI 54880

USA

E-mail address: xgu@uwsuper.edu

HONG-JiAn LAI

Department of Mathematics

WEST Virginia UNIVERSITY

Morgantown, WV 26506

USA

E-mail address: hongjianlai@gmail.com

ReCeived July 12, 2013 